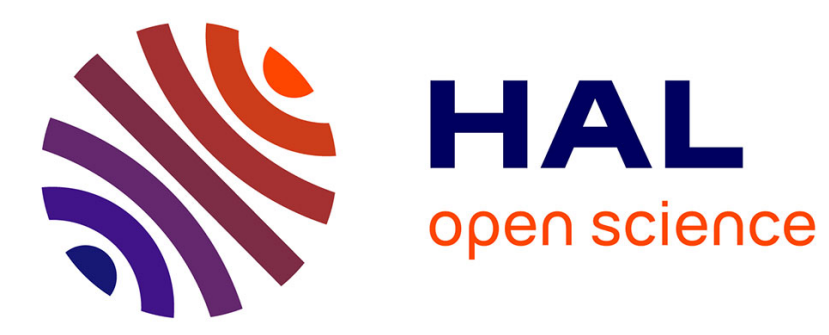

\title{
On the use of the wave finite element method for passive vibration control of periodic structures
}

Priscilla Brandao Silva, Jean-Mathieu Mencik, Roberto Arruda

\section{To cite this version:}

Priscilla Brandao Silva, Jean-Mathieu Mencik, Roberto Arruda. On the use of the wave finite element method for passive vibration control of periodic structures. Advances in Aircraft and Spacecraft Science, 2016, 3 (3), pp.299-315. 10.12989/aas.2016.3.3.299 . hal-01339068

\section{HAL Id: hal-01339068 \\ https://hal.science/hal-01339068}

Submitted on 29 Jun 2016

HAL is a multi-disciplinary open access archive for the deposit and dissemination of scientific research documents, whether they are published or not. The documents may come from teaching and research institutions in France or abroad, or from public or private research centers.
L'archive ouverte pluridisciplinaire HAL, est destinée au dépôt et à la diffusion de documents scientifiques de niveau recherche, publiés ou non, émanant des établissements d'enseignement et de recherche français ou étrangers, des laboratoires publics ou privés. 


\title{
On the use of the wave finite element method for passive vibration control of periodic structures
}

\author{
Priscilla B. Silva ${ }^{* 1}$, Jean-Mathieu Mencik ${ }^{2 a}$ and José R. F. Arruda ${ }^{1 b}$ \\ ${ }^{1}$ Departamento de Mecânica Computacional, Faculdade de Engenharia Mecânica, Universidade Estadual de \\ Campinas, R. Mendeleyev, 200, 13083-860, Campinas-SP, Brazil \\ ${ }^{2}$ INSA Centre Val de Loire, Université François Rabelais de Tours, LMR EA 2640, Campus de Blois, 3 rue de \\ la chocolaterie, CS 23410, 41034 Blois Cedex, France
}

(Received May 29, 2015, Revised July 10, 2015, Accepted July 17, 2015)

\begin{abstract}
In this work, a strategy for passive vibration control of periodic structures is proposed which involves adding a periodic array of simple resonant devices for creating band gaps. It is shown that such band gaps can be generated at low frequencies as opposed to the well known Bragg scattering effects when the wavelengths have to meet the length of the elementary cell of a periodic structure. For computational purposes, the wave finite element (WFE) method is investigated, which provides a straightforward and fast numerical means for identifying band gaps through the analysis of dispersion curves. Also, the WFE method constitutes an efficient and fast numerical means for analyzing the impact of band gaps in the attenuation of the frequency response functions of periodic structures. In order to highlight the relevance of the proposed approach, numerical experiments are carried out on a 1D academic rod and a 3D aircraft fuselage-like structure.
\end{abstract}

Keywords: wave finite element method; periodic structures; band gaps; passive vibration control; aircraft fuselage

\section{Introduction}

The development of passive vibration control strategies of industrial periodic structures, which use band gaps, is a current research topic which aims at proposing efficient lightweight solutions for global reduction of the vibration levels. Actually, there exist two band gap mechanisms which are either of Bragg scattering type (Sigalas and Economou 1992, Kushwaha et al. 1993, Kushwaha et al. 1994), or locally resonant type (Liu et al. 2000, Goffaux et al. 2002). Regarding Bragg scattering, band gaps represent zones of destructive interference between incident and reflected waves which occur when the wavelengths are of the same order of magnitude as the dimensions of the periodic cells. This means that, at low frequencies, such band gaps only occur in large-sized structures. In elastodynamics, this mechanism has been highlighted in numerous engineering 
systems, which are usually named phononic crystals, such as rods, plates and cylindrical shells (Bennett and Accorsi 1994, Lee et al. 2010, Sorokin and Ershova 2004, Goldstein et al. 2011). More recently, researchers in the field have moved their attention to locally resonant devices which are motivated by the possibility of designing band gaps at low frequencies, i.e., in case when the wavelengths exceed the dimensions of the periodic cells (Liu et al. 2000). One can mention for instance locally resonant phononic crystals, also known as metamaterials, which exhibit effective negative elastic constants in certain frequency bands. The key idea here is to consider periodic arrays of locally resonant devices which are attached to periodic structures. Although the concept has been applied to academic structures such as strings, rods, beams, plates (Xiao et al. 2011, Xiao et al. 2012, Xiao et al. 2013, Wang et al. 2013), its application to complex structures with true periodicities, such as aircraft fuselages, seems to constitute an open research topic.

Analyzing the feasibility of using periodic arrays of locally resonant devices for band gaps generation and passive vibration control of periodic structures is the motivation of the present work. For this purpose, the wave finite element (WFE) method is used, which provides an efficient means for computing the dispersion curves of the waves traveling along periodic structures (Zhong and Williams 1995, Mencik and Ichchou 2005). Also, the WFE method is used for computing the forced response of complex periodic structures, at a low computational cost (Mencik 2014, Silva et al. 2015).

The rest of the paper is organized as follows. In Section 2, the basics of the WFE method are recalled regarding the calculation of wave modes in periodic structures. Also, the strategy for computing the dispersion curves and the frequency response functions (FRFs) of periodic structures are presented. In Section 3, a comprehensive numerical analysis is carried out regarding a 1D homogeneous rod which is connected to periodic arrays of harmonic oscillators, or a single vibration absorbers. Additional numerical experiments are finally brought in Section 4 regarding a 3D fuselage-like structure equipped with a periodic array of locally resonant devices.

\section{Modeling of periodic structures}

\subsection{WFE method}

The WFE method focuses on calculating wave modes traveling along periodic structures, and further, the forced response of those structures by means of wave-based matrix systems of small size, which can be computed in a very fast way. Those structures are periodic in the sense that their FE mesh is composed of identical substructures/cells (with a length of $\Delta$ ) which are assembled to each other along a certain straight direction (say, axis $x$ ), see Fig. 1. The computation of the wave modes involves considering the FE model of one single substructure (Mencik and Ichchou 2005). One of the advantages of this numerical approach is that, as opposed to analytical approaches, periodic structures composed of complex and heterogeneous substructures can be taken into account.

Within the framework of band gap analysis, a periodic structure is designed so that each substructure contains a locally resonant device, as shown in Fig. 1. By considering the FE model of one substructure, a dynamic equilibrium equation is expressed in matrix form, as follows

$$
\left[-\omega^{2} \mathbf{M}+(1+\mathrm{i} \eta) \mathbf{K}\right] \mathbf{q}=\mathbf{F},
$$

where $\omega$ is the angular frequency; $\mathbf{M}, \mathbf{K}$ and $\eta$ are the mass matrix, stiffness matrix and loss factor 


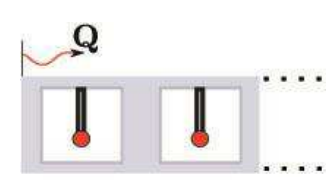

(1)
(2)

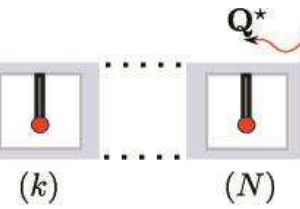

(a)

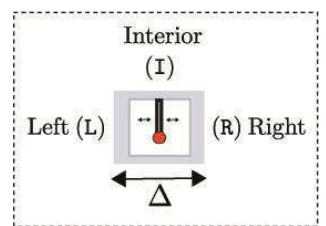

(b)

Fig. 1 Schematics of a one-dimensional periodic structure composed of $N$ substructures (a) and of a particular substructure (b)

of the substructure, respectively; $\mathbf{q}$ and $\mathbf{F}$ are the vectors of nodal displacements/rotations and forces/moments of the substructure, respectively. By partitioning the degrees of freedom (DOFs) into those on the left boundary $\mathrm{L}$ and right boundary $\mathrm{R}$ of the substructure, and internal DOFs I, this yields

$$
\left[\begin{array}{ccc}
\mathbf{D}_{\mathrm{LL}} & \mathbf{D}_{\mathrm{LI}} & \mathbf{D}_{\mathrm{LR}} \\
\mathbf{D}_{\mathrm{IL}} & \mathbf{D}_{\mathrm{II}} & \mathbf{D}_{\mathrm{IR}} \\
\mathbf{D}_{\mathrm{RL}} & \mathbf{D}_{\mathrm{RI}} & \mathbf{D}_{\mathrm{RR}}
\end{array}\right]\left[\begin{array}{c}
\mathbf{q}_{\mathrm{L}} \\
\mathbf{q}_{\mathrm{I}} \\
\mathbf{q}_{\mathrm{R}}
\end{array}\right]=\left[\begin{array}{c}
\mathbf{F}_{\mathrm{L}} \\
\mathbf{0} \\
\mathbf{F}_{\mathrm{R}}
\end{array}\right],
$$

where $\mathbf{D}=-\omega^{2} \mathbf{M}+(1+\mathrm{i} \eta) \mathbf{K}$ is the dynamic stiffness matrix of the substructure. Notice that $\mathbf{F}_{\mathrm{I}}=\mathbf{0}$ because the internal DOFs are assumed to be free from excitations. By condensing the matrix $\mathbf{D}$ on the left and right boundaries of the substructure, Eq. (2) leads to

$$
\left[\begin{array}{ll}
\mathbf{D}_{\mathrm{LL}}^{*} & \mathbf{D}_{\mathrm{LR}}^{*} \\
\mathbf{D}_{\mathrm{RL}}^{*} & \mathbf{D}_{\mathrm{RR}}^{*}
\end{array}\right]\left[\begin{array}{l}
\mathbf{q}_{\mathrm{L}} \\
\mathbf{q}_{\mathrm{R}}
\end{array}\right]=\left[\begin{array}{l}
\mathbf{F}_{\mathrm{L}} \\
\mathbf{F}_{\mathrm{R}}
\end{array}\right],
$$

where $\mathbf{D}^{*}=\mathbf{D}_{\mathrm{BB}}-\mathbf{D}_{\mathrm{BI}} \mathbf{D}_{\mathrm{II}}^{-1} \mathbf{D}_{\mathrm{IB}}$ is the condensed dynamic stiffness matrix of the substructure $(B$ denoting the DOFs on the left and right boundaries). It is worth pointing out that the left and right boundaries of the substructures are meshed in the same way, i.e., with the same number $n$ of DOFs. This particularly means that the vectors $\mathbf{q}_{\mathrm{L}}, \mathbf{q}_{\mathrm{R}}, \mathbf{F}_{\mathrm{L}}$ and $\mathbf{F}_{\mathrm{R}}$ have the same size, i.e, $n \times 1$. By considering two consecutive substructures $k-1$ and $k$, a state vector representation can be proposed as follows

$$
\mathbf{u}_{\mathrm{L}}^{(k)}=\mathbf{S} \mathbf{u}_{\mathrm{L}}^{(k-1)} \quad, \quad \mathbf{u}_{\mathrm{R}}^{(k)}=\mathbf{S u}_{\mathrm{R}}^{(k-1)},
$$

where $\mathbf{u}_{\mathrm{L}}=\left[\begin{array}{ll}\mathbf{q}_{\mathrm{L}}^{T} & -\mathbf{F}_{\mathrm{L}}^{T}\end{array}\right]^{T}$ and $\mathbf{u}_{\mathrm{R}}=\left[\begin{array}{ll}\mathbf{q}_{\mathrm{R}}^{T} & \mathbf{F}_{\mathrm{R}}^{T}\end{array}\right]^{T}$ are $2 n \times 1$ state vectors. Also, $\mathbf{S}$ is a $2 n \times 2 n$ symplectic transfer matrix expressed by

$$
\mathbf{S}=\left[\begin{array}{cc}
-\mathbf{D}_{\mathrm{LR}}^{*-1} \mathbf{D}_{\mathrm{LL}}^{*} & \mathbf{D}_{\mathrm{LR}}^{*-1} \\
\mathbf{D}_{\mathrm{RL}}^{*}-\mathbf{D}_{\mathrm{RR}}^{*} \mathbf{D}_{\mathrm{LR}}^{*-1} \mathbf{D}_{\mathrm{LL}}^{*} & -\mathbf{D}_{\mathrm{RR}}^{*} \mathbf{D}_{\mathrm{LR}}^{*-1}
\end{array}\right] .
$$

According to Bloch's theorem, the eigensolutions of the matrix S- i.e., eigenvalues $\mu_{j}$ and right eigenvectors $\phi_{j}^{-}$are referred to as waves traveling along the periodic structure. More precisely, what states Bloch's theorem is that the eigenvalues are expressed as $\mu_{j}=e^{-\mathrm{i} \beta_{j} \Delta}$ where $\beta_{j}$ has the meaning of wavenumbers, while the eigenvectors $\phi_{j}$ are to be understood as wave shapes. The 
eigenproblem which needs to be solved for expressing $\mu_{j}$ and $\phi_{j}$ is written as follows

$$
\mathbf{S} \phi_{j}=\mu_{j} \phi_{j}
$$

Notice that the wave shapes $\phi_{j}$ are to be partitioned into $n \times 1$ vectors of displacements/rotations $\phi_{\mathrm{g} j}$ and forces/moments $\phi_{\mathrm{F} j}$, which provide spatial descriptions for the wave motion over the substructure boundaries. The eigensolutions $\left(\mu_{j}, \phi_{j}\right)$ are referred to as the wave modes of the structure. In fact, there are twice as many wave modes as the number of DOFs used to discretize each substructure boundary. Due to the symplectic property of the matrix $\mathbf{S}$, the eigensolutions appears in pairs as $\left(\mu_{j}, 1 / \mu_{j}\right)$, which correspond to $n$ right-going and $n$ left-going wave modes. As some damping is always present in real systems, the magnitudes of the eigenvalues $\mu_{j}$ are different from one. Hence the wave modes can be classified into right-going modes for which the magnitudes of $\mu_{j}$ are less than one, and left-going modes for which the magnitudes of $\mu_{j}$ are greater than one. By convention, those left-going and right-going wave modes are denoted as $\left\{\left(\mu_{j}, \phi_{j}\right)\right\}_{j=1, \ldots, n}$ and $\left\{\left(\mu_{j}^{\prime}, \phi_{j}^{\prime}\right)\right\}_{j=1, \ldots, n}$, where

$$
\mu_{j}^{\prime}=1 / \mu_{j} \quad \forall j \in\{1, \ldots, n\} .
$$

Also, there exist analytical relations which link the wave shapes $\phi_{j}^{\prime}$ and $\phi_{j}$ as follows

$$
\phi_{\mathrm{q} j}^{\prime}=\mathbf{R} \phi_{\mathrm{q} j} \quad, \quad \phi_{\mathrm{F} j}^{\prime}=-\mathbf{R} \phi_{\mathrm{F} j} \quad \forall j \in\{1, \ldots, n\},
$$

where $\mathbf{R}$ relates a symmetry transformation matrix (Mencik 2010). Notice that Eq. (8) is true provided that the periodic structure is composed of symmetric substructures.

\subsection{Dispersion curves}

Within the WFE framework, the dispersion curve of each wave mode is obtained by analyzing the frequency evolution of the wavenumbers $\beta_{j}$ which are defined so that $\mu_{j}=e^{-\mathrm{i} \beta_{j} \Delta}, \mu_{j}$ and $\Delta$ being the eigenvalues of the matrix $\mathbf{S}$ (Eq. (5)) and the substructure length, respectively. Tracking each wave mode over the frequency domain appears to be a crucial task aiming at providing a deep physical insight of the dynamic behavior of periodic structures. Indeed, it will be shown later in this paper that the dispersion curves can be used to predict the far-field dynamic behavior of periodic structures with a large number of substructures. The issue when assessing the frequency evolution of $\beta_{j}$ (or $\mu_{j}$ ) consists in tracking each wave mode over the frequency domain, it being understood that many wave modes are to be computed at several discrete frequencies and there exists a priori no direct connection to link these modes between two consecutive frequencies. In other words, a given mode $m$ defined at a frequency $\omega_{i}$ may not match with the mode $m$ defined at the previous frequency $\omega_{i-1}$.

The wave mode tracking procedure that is considered here makes use of the symplectic orthogonality properties of the wave modes (Zhong and Williams 1995) between two consecutive discrete frequencies $\omega_{i-1}$ and $\omega_{i}$ spaced by a small step $\Delta \omega$. The procedure can be stated as follows (Mencik 2010). Given two wave modes $l$ and $m$ verifying $\mu_{l}=1 / \mu_{m}$ at the frequency $\omega_{i-1}$, the wave mode $m$ at the frequency $\omega_{i}$ is chosen so that

$$
\left|\frac{\phi_{l}\left(\omega_{i-1}\right)^{T}}{\| \phi_{l}\left(\omega_{i-1}\right) \mid} \mathbf{J} \frac{\phi_{m}\left(\omega_{i}\right)}{\left\|\phi_{m}\left(\omega_{i}\right)\right\|}\right|=\max _{k}\left\{\left|\frac{\phi_{l}\left(\omega_{i-1}\right)^{T}}{\| \phi_{l}\left(\omega_{i-1}\right) \mid} \mathbf{J} \frac{\phi_{k}\left(\omega_{i}\right)}{\left\|\phi_{k}\left(\omega_{i}\right)\right\|}\right|\right\}
$$


where

$$
\mathbf{J}=\left[\begin{array}{cc}
\mathbf{0}_{n} & \mathbf{I}_{n} \\
-\mathbf{I}_{n} & \mathbf{0}_{n}
\end{array}\right]
$$

\subsection{Forced response computation}

Predicting the frequency behavior of a bounded periodic structure, composed of $N$ substructures and subjected to arbitrary boundary conditions on its left and right ends, consists in expanding the vectors of displacements/rotations and forces/moments, on the left or right boundary of a substructure $k$, using wave bases $\left\{\phi_{\mathrm{q} j}\right\}_{j=1, \cdots, n} \cup\left\{\phi_{\mathrm{q} j}^{\prime}\right\}_{j=1, \cdots, n}$ and $\left\{\phi_{\mathrm{F} j}\right\}_{j=1, \cdots, n} \cup\left\{\phi_{\mathrm{F} j}^{\prime}\right\}_{j=1, \cdots, n}$ (Mencik 2014)

$$
\begin{array}{rll}
\mathbf{q}_{\mathrm{L}}^{(k)} & =\boldsymbol{\Phi}_{\mathrm{q}} \boldsymbol{\mu}^{k-1} \mathbf{Q}+\boldsymbol{\Phi}_{\mathrm{q}}^{\prime} \boldsymbol{\mu}^{N-k+1} \mathbf{Q}^{\prime} \quad, \quad \mathbf{q}_{\mathrm{R}}^{(k)}=\boldsymbol{\Phi}_{\mathrm{q}} \boldsymbol{\mu}^{k} \mathbf{Q}+\boldsymbol{\Phi}_{\mathrm{q}}^{\prime} \boldsymbol{\mu}^{N-k} \mathbf{Q}^{\prime} \quad k=1, \ldots, N, \\
-\mathbf{F}_{\mathrm{L}}^{(k)}=\boldsymbol{\Phi}_{\mathrm{F}} \boldsymbol{\mu}^{k-1} \mathbf{Q}+\boldsymbol{\Phi}_{\mathrm{F}}^{\prime} \boldsymbol{\mu}^{N-k+1} \mathbf{Q}^{\prime}, & \mathbf{F}_{\mathrm{R}}^{(k)}=\boldsymbol{\Phi}_{\mathrm{F}} \boldsymbol{\mu}^{k} \mathbf{Q}+\boldsymbol{\Phi}_{\mathrm{F}}^{\prime} \boldsymbol{\mu}^{N-k} \mathbf{Q}^{\prime} & k=1, \ldots, N,
\end{array}
$$

where $\boldsymbol{\Phi}_{\mathrm{q}}, \quad \boldsymbol{\Phi}_{\mathrm{q}}^{\prime}, \quad \boldsymbol{\Phi}_{\mathrm{F}}$ and $\boldsymbol{\Phi}_{\mathrm{F}}^{\prime}$ are $n \times n$ matrices expressed as $\boldsymbol{\Phi}_{\mathrm{q}}=\left\lfloor\begin{array}{llll}\phi_{\mathrm{q} 1} & \cdots & \phi_{\mathrm{q} n}\end{array}\right\rfloor$, $\boldsymbol{\Phi}_{\mathrm{q}}^{\prime}=\left[\begin{array}{lll}\phi_{\mathrm{q} 1}^{\prime} & \cdots & \phi_{\mathrm{q} n}^{\prime}\end{array}\right] \quad, \quad \boldsymbol{\Phi}_{\mathrm{F}}=\left[\begin{array}{lll}\phi_{\mathrm{F} 1} & \cdots & \phi_{\mathrm{F} n}\end{array}\right] \quad$ and $\quad \boldsymbol{\Phi}_{\mathrm{F}}^{\prime}=\left[\begin{array}{lll}\phi_{\mathrm{F} 1}^{\prime} & \cdots & \phi_{\mathrm{F} n}^{\prime}\end{array}\right] \quad . \quad$ Also, $\mathbf{Q}=\left[\begin{array}{lll}Q_{1} & \cdots & Q_{n}\end{array}\right]^{T}$ and $\mathbf{Q}^{\prime}=\left[\begin{array}{lll}Q_{1}^{\prime} & \cdots & Q_{n}^{\prime}\end{array}\right]^{T}$ denote the vectors of wave amplitudes at the left and right ends of the whole periodic structure, respectively. Finally, $\boldsymbol{\mu}$ is the $n \times n$ diagonal matrix defined so that $\boldsymbol{\mu}=\operatorname{diag}\left\{\mu_{j}\right\}_{j=1, \ldots, n}$ where it is understood that the magnitudes $\left|\mu_{j}\right|$ are less than one (see above Eq. (7)).

By considering Eqs. (11) and (12), the condensed dynamic stiffness matrix of the whole periodic structure can be expressed in a straightforward way, as follows. Denote as $\mathbf{D}^{(s)}$ the dynamic stiffness matrix of the periodic structure which is condensed on its left and the right ends. Thus, the dynamic equilibrium equation of the periodic structure can be written as follows

$$
\mathbf{D}^{(s)}\left[\begin{array}{c}
\mathbf{q}_{\mathrm{L}}^{(1)} \\
\mathbf{q}_{\mathrm{R}}^{(N)}
\end{array}\right]=\left[\begin{array}{c}
\mathbf{F}_{\mathrm{L}}^{(1)} \\
\mathbf{F}_{\mathrm{R}}^{(N)}
\end{array}\right] .
$$

In order to express the dynamic stiffness matrix $\mathbf{D}^{(s)}$, the vectors of nodal displacements/rotations $\left(\mathbf{q}_{\mathrm{L}}^{(1)}, \mathbf{q}_{\mathrm{R}}^{(N)}\right)$ and forces/moments $\left(\mathbf{F}_{\mathrm{L}}^{(1)}, \mathbf{F}_{\mathrm{R}}^{(N)}\right)$ on the left and right ends of the periodic structure are expressed by means of Eqs. (11) and (12) as follows

$$
\left[\begin{array}{c}
\mathbf{q}_{\mathrm{L}}^{(1)} \\
\mathbf{q}_{\mathrm{R}}^{(N)}
\end{array}\right]=\left[\begin{array}{cc}
\boldsymbol{\Phi}_{\mathrm{q}} & \boldsymbol{\Phi}_{\mathrm{q}}^{\prime} \boldsymbol{\mu}^{N} \\
\boldsymbol{\Phi}_{\mathrm{q}} \boldsymbol{\mu}^{N} & \boldsymbol{\Phi}_{\mathrm{q}}^{\prime}
\end{array}\right]\left[\begin{array}{c}
\mathbf{Q} \\
\mathbf{Q}^{\prime}
\end{array}\right],
$$

and

$$
\left[\begin{array}{c}
\mathbf{F}_{\mathrm{L}}^{(1)} \\
\mathbf{F}_{\mathrm{R}}^{(N)}
\end{array}\right]=\left[\begin{array}{cc}
-\boldsymbol{\Phi}_{\mathrm{F}} & -\boldsymbol{\Phi}_{\mathrm{F}}^{\prime} \boldsymbol{\mu}^{N} \\
\boldsymbol{\Phi}_{\mathrm{F}} \boldsymbol{\mu}^{N} & \boldsymbol{\Phi}_{\mathrm{F}}^{\prime}
\end{array}\right]\left[\begin{array}{c}
\mathbf{Q} \\
\mathbf{Q}^{\prime}
\end{array}\right] .
$$


To derive the condensed dynamic stiffness matrix $\mathbf{D}^{(s)}$ of the periodic structure, Eq. (14) is left multiplied by the following matrix

$$
\left[\begin{array}{cc}
\boldsymbol{\Phi}_{\mathrm{q}}^{-1} & \mathbf{0} \\
\mathbf{0} & \boldsymbol{\Phi}_{\mathrm{q}}^{\prime-1}
\end{array}\right]
$$

which yields

$$
\left[\begin{array}{cc}
\boldsymbol{\Phi}_{\mathrm{q}}^{-1} & \mathbf{0} \\
\mathbf{0} & \boldsymbol{\Phi}_{\mathrm{q}}^{\prime-1}
\end{array}\right]\left[\begin{array}{c}
\mathbf{q}_{\mathrm{L}}^{(1)} \\
\mathbf{q}_{\mathrm{R}}^{(N)}
\end{array}\right]=\left[\begin{array}{cc}
\mathbf{I} & \boldsymbol{\Phi}_{\mathrm{q}}^{-1} \boldsymbol{\Phi}_{\mathrm{q}}^{\prime} \boldsymbol{\mu}^{N} \\
\boldsymbol{\Phi}_{\mathrm{q}}^{\prime-1} \boldsymbol{\Phi}_{\mathrm{q}} \boldsymbol{\mu}^{N} & \mathbf{I}
\end{array}\right]\left[\begin{array}{c}
\mathbf{Q} \\
\mathbf{Q}^{*}
\end{array}\right]
$$

The motivation behind the use of the matrix (16) is to make the matrix occurring on the right hand side of Eq. (17) well-conditioned, as explained in (Mencik 2010). As it turns out, the vectors of wave amplitudes $\mathbf{Q}$ and $\mathbf{Q}^{\prime}$ can be expressed from Eq. (17) as

$$
\left[\begin{array}{c}
\mathbf{Q} \\
\mathbf{Q}^{*}
\end{array}\right]=\left[\begin{array}{cc}
\mathbf{I} & \boldsymbol{\Phi}_{\mathrm{q}}^{-1} \boldsymbol{\Phi}_{\mathrm{q}}^{*} \boldsymbol{\mu}^{N} \\
\boldsymbol{\Phi}_{\mathrm{q}}^{\prime-1} \boldsymbol{\Phi}_{\mathrm{q}} \boldsymbol{\mu}^{N} & \mathbf{I}
\end{array}\right]^{-1}\left[\begin{array}{cc}
\boldsymbol{\Phi}_{\mathrm{q}}^{-1} & \mathbf{0} \\
\mathbf{0} & \boldsymbol{\Phi}_{\mathrm{q}}^{\prime-1}
\end{array}\right]\left[\begin{array}{c}
\mathbf{q}_{\mathrm{L}}^{(1)} \\
\mathbf{q}_{\mathrm{R}}^{(N)}
\end{array}\right] .
$$

The condensed dynamic stiffness matrix $\mathbf{D}^{(s)}$ of the periodic structure, Eq. (13), is readily derived from Eqs. (15) and (18), as follows

$$
\mathbf{D}^{(s)}=\left[\begin{array}{cc}
-\boldsymbol{\Phi}_{\mathrm{F}} & -\boldsymbol{\Phi}_{\mathrm{F}}^{\prime} \boldsymbol{\mu}^{N} \\
\boldsymbol{\Phi}_{\mathrm{F}} \boldsymbol{\mu}^{N} & \boldsymbol{\Phi}_{\mathrm{F}}^{\prime}
\end{array}\right]\left[\begin{array}{cc}
\mathbf{I} & \boldsymbol{\Phi}_{\mathrm{q}}^{-1} \boldsymbol{\Phi}_{\mathrm{q}}^{\prime} \boldsymbol{\mu}^{N} \\
\boldsymbol{\Phi}_{\mathrm{q}}^{\prime-1} \boldsymbol{\Phi}_{\mathrm{q}} \boldsymbol{\mu}^{N} & \mathbf{I}
\end{array}\right]^{-1}\left[\begin{array}{cc}
\boldsymbol{\Phi}_{\mathrm{q}}^{-1} & \mathbf{0} \\
\mathbf{0} & \boldsymbol{\Phi}_{\mathrm{q}}^{\prime-1}
\end{array}\right] .
$$

The determination of the vector of displacements/rotations $\left[\begin{array}{ll}\mathbf{q}_{\mathrm{L}}^{(1) T} & \mathbf{q}_{\mathrm{R}}^{(N) T}\end{array}\right]^{T}$ can be achieved in the same way as in the conventional FE procedure, i.e., by considering the inverse of the matrix $\mathbf{D}^{(s)}$, see Eq. (13). It is worth emphasizing that the consideration of assemblies composed of several periodic structures and other FE components, that may be connected to each other in arbitrary ways, can be addressed without any additional issues through FE procedures (Silva et al. 2014, 2015).

\section{Comparisons between local and global attenuation effects}

Vibration absorbers are mass-spring or mass-spring-damper systems which are widely used in the field of vibration control engineering. Since the early development of the theory of dynamic vibration absorbers (Ormondroyd and den Hartog 1928), they have been applied in a wide range of applications. In many situations, a single vibration absorber punctually attached to one structure is considered so as to avoid the vibrations induced by some vibrational modes. The use of periodic arrays of these locally resonant devices, along structures, is very recent (Thompson 2008). It has been first investigated by Kashina and Tyutekin (1990) who have proposed the use of a set of undamped resonators to reduce the longitudinal and flexural motions in beams and plates. This idea has received new attention with the publication of the work of Liu et al. (2000), in which emphasis is placed on exploring the impact of using periodic arrays of resonant devices on wave 
propagation in artificial periodic composites known as phononic crystals. In such systems, band gaps can be optimized by tuning the resonator's natural frequency to the control procedure's targeted one.

In this section, the decrease of the vibration levels produced by adding a periodic array of resonant devices to a 1D homogeneous rod is analyzed by means of the WFE method. The efficiency in using such devices is highlighted through comparisons with the vibration levels produced by a single vibration absorber. While a single vibration absorber produces local vibration attenuation, a periodic array of small absorbers yields global vibration control. These phenomena are illustrated here regarding a simple 1D homogeneous rod structure having longitudinal vibrations (see Fig. 2), whose material and geometric properties are listed in Table 1. The left and right ends of the rod are, respectively, excited by a unitary axial harmonic force, and free. Here, the structure is either connected to a periodic array of nine spring-mass systems (Fig. 2(b)), or connected to one single spring-mass system (Fig. 2(c)). The dispersion curves and the FRFs of the uncoupled and coupled structure are computed using the WFE method. Within this framework, the substructure being considered (Section 2.1) represents either a single rod cell, or a rod cell with a spring-mass resonant system (see Fig. 2(b)). Notice that each substructure is modelled by means of the spectral element method (SEM) which provides an exact analytical expression of the condensed dynamic stiffness matrix $\mathbf{D}^{*}$ (Eq. (3)) (Doyle 1997, Xiao et al. 2012). Within the analytical framework, the displacement field of a rod cell is described in a standard way by means of two longitudinal waves, i.e., two propagating ones traveling in opposite directions along a onedimensional system. The consideration of mass-spring-damper systems is simply achieved through basic kinematic compatibility and force equilibrium equation.

Band gaps formed by Bragg scattering mechanisms occur in periodic structures when the wavelength of a traveling wave is equal to twice the substructure length $\Delta$ (Sigalas et al. 2005). The corresponding frequency $f_{\text {ref }}$, which is the frequency-edge of the first Bragg scattering band gap, in the case of longitudinal wave propagation is given by

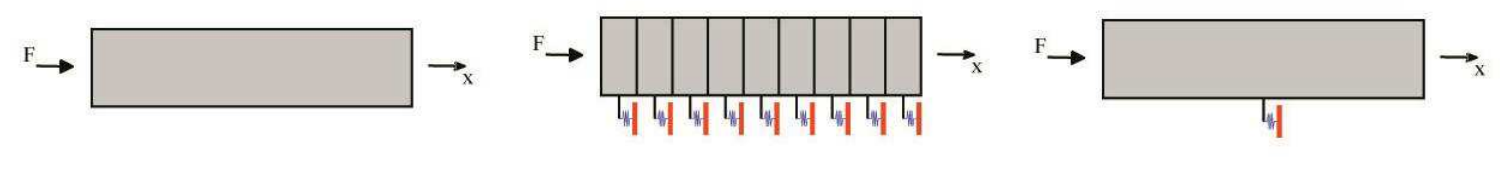

(a)

(b)

(c)

Fig. 2 1D homogeneous rod: (a) without resonant devices; (b) with a periodic array of resonant devices; (c) with a single vibration absorber

Table 1 Characteristics of the 1D homogeneous rod

\begin{tabular}{cc}
\hline Parameter & Value \\
\hline Density $(\rho)$ & $1200 \mathrm{~kg} / \mathrm{m}^{3}$ \\
Young's Modulus $(E)$ & $3 \mathrm{GPa}$ \\
Poisson's ratio $(v)$ & 0.3 \\
Structural damping $(\eta)$ & 0.01 \\
Cross-section area $(Q)$ & $10010^{-6} \mathrm{~m}^{2}$ \\
Total length $(L)$ & $4.5 \mathrm{~m}$ \\
\hline
\end{tabular}




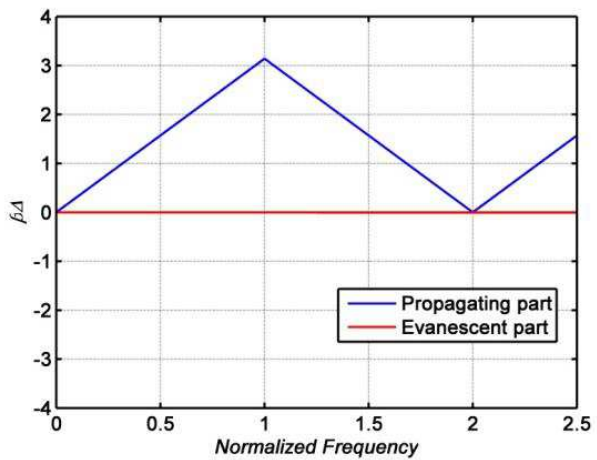

substructure

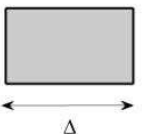

(a)

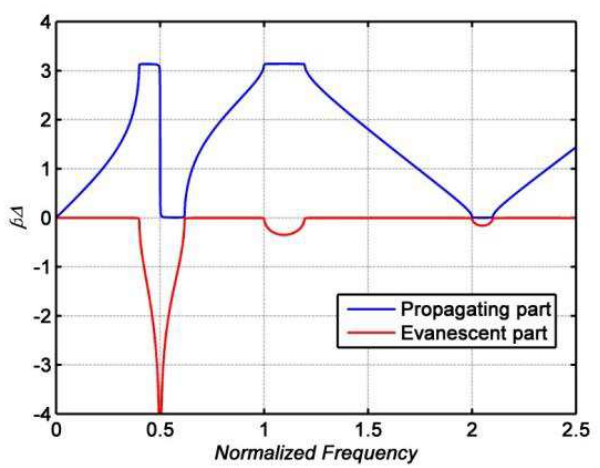

substructure

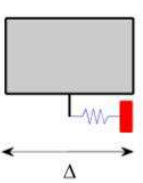

(b)

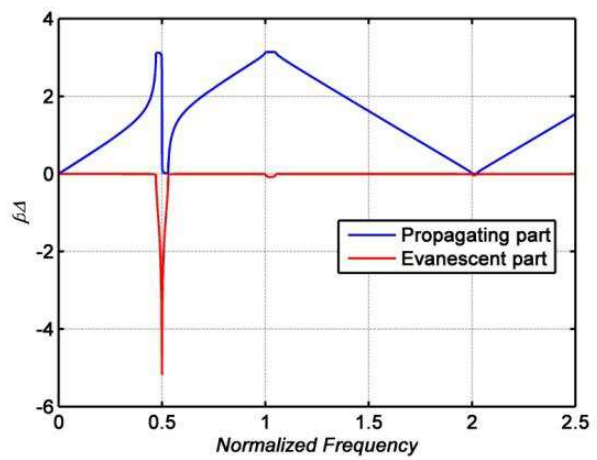

substructure

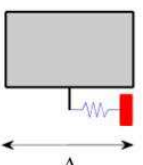

(c)

Fig. 3 Dispersion curves of the longitudinal wave in the homogeneous rod: (a) without resonant devices; (b) with a periodic array of resonant devices composed of 9 resonators with a mass ratio of $44 \%$; (c) with a periodic array of resonant devices composed of 9 resonators with a mass ratio of $14 \%$. (blue color) real part of $\beta \Delta$; (red color) imaginary part of $\beta \Delta$

$$
f_{\text {ref }}=\frac{1}{2 \Delta} \sqrt{\frac{E}{\rho}} .
$$

To begin with, a periodic array of nine resonant devices (Fig. 2(b)) is considered so that their 
resonance frequency is $0.5 f_{\text {ref }}=790.6 \mathrm{~Hz}$. Two test cases are considered which involve resonant devices whose total mass represents, respectively, $44 \%$ and $14 \%$ of that of the rod with the resonant devices. The related dispersion curves of the longitudinal wave traveling in the coupled rod are displayed in Fig. 3, along with the dispersion curve that concerns the uncoupled rod. Here the non-dimensional wavenumber $\beta \Delta$ is analyzed as a function of the non-dimensional frequency $f / f_{\text {ref }}$, where $f=2 \pi \omega$. As it can be seen in Fig. 3(a), the longitudinal wave in the rod without resonant devices is always propagative, as expected. Notice that the imaginary part of $\beta \Delta$, although small, is not equal to zero due to damping effects. Regarding the resonant devices, three band gaps (i.e., attenuation zones) occur in Figs. 3(b)-(c): one is around $0.5 f_{\text {ref }}$ while others are around $f_{\text {ref }}$ and 2 $f_{\text {ref. }}$. It is seen that the first band gap occurs at the resonance frequency of the spring-mass system, which is a characteristic of band gaps formed by local resonance mechanisms. On the other hand, other band gaps occur at multiples of the reference frequency and have constant phase angle, which characterize them as band gaps of Bragg-type.

To highlight further those band gaps, the 2D maps of the FRFs of the rod are shown in Fig. 4. In these figures, the FRFs are plotted as functions of the abscissa $x$ along the length of the rod. Regarding Fig. 4(a), the resonance frequencies of the rod are clearly identified by red lines. Attenuation zones occur in Figs. 4(b)-(c) in accordance with the band gaps in Figs. 3(b)-(c), which extend along the length of the structure except at $x=0 \mathrm{~m}$, i.e., in the vicinity of the excitation. The
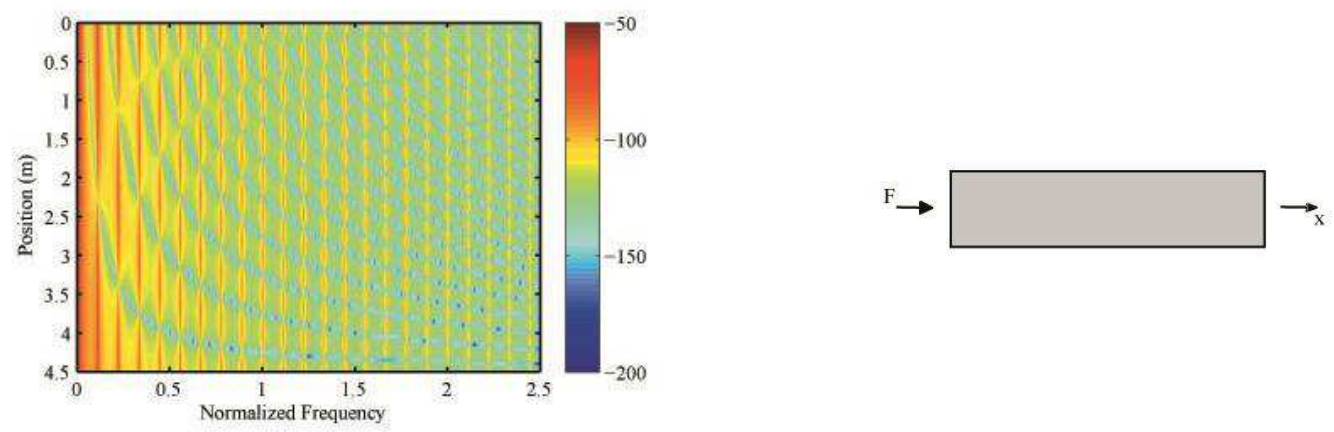

(a)
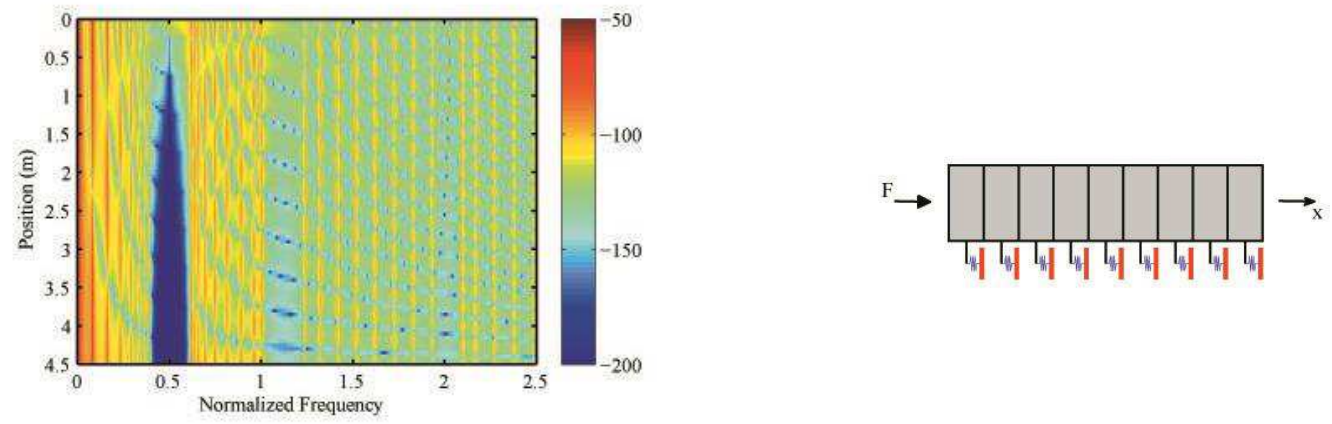

(b)

Fig. 4 2D maps of the FRF (in $\mathrm{dB}$ ) of the homogeneous rod: (a) without resonant devices; (b) with a periodic array of resonant devices composed of 9 resonators with a mass ratio of $44 \%$; (c) with a periodic array of resonant devices composed of 9 resonators with a mass ratio of $14 \%$ 

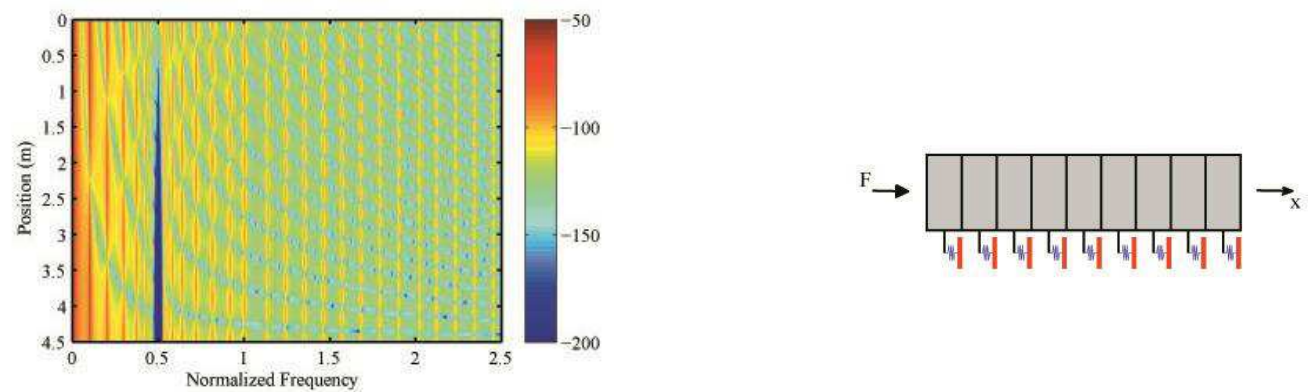

(c)

Fig. 4 Continued
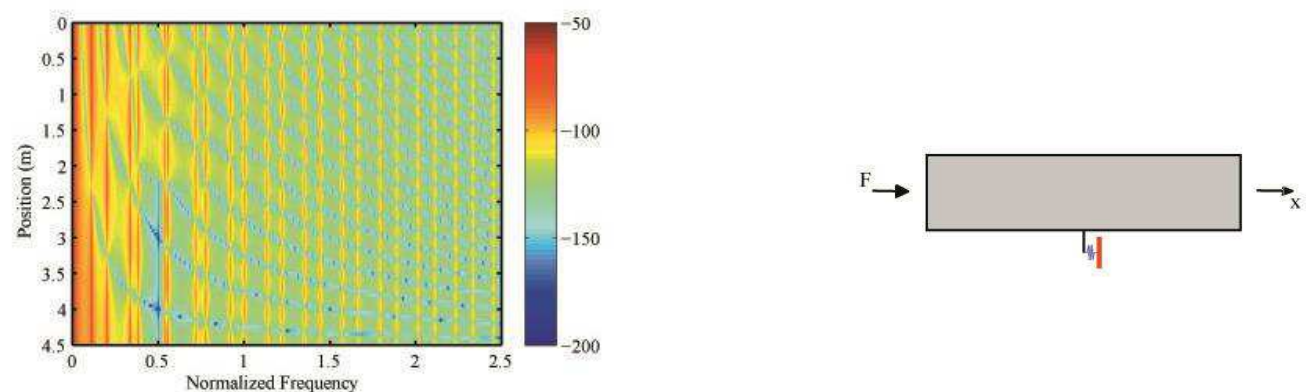

(a)
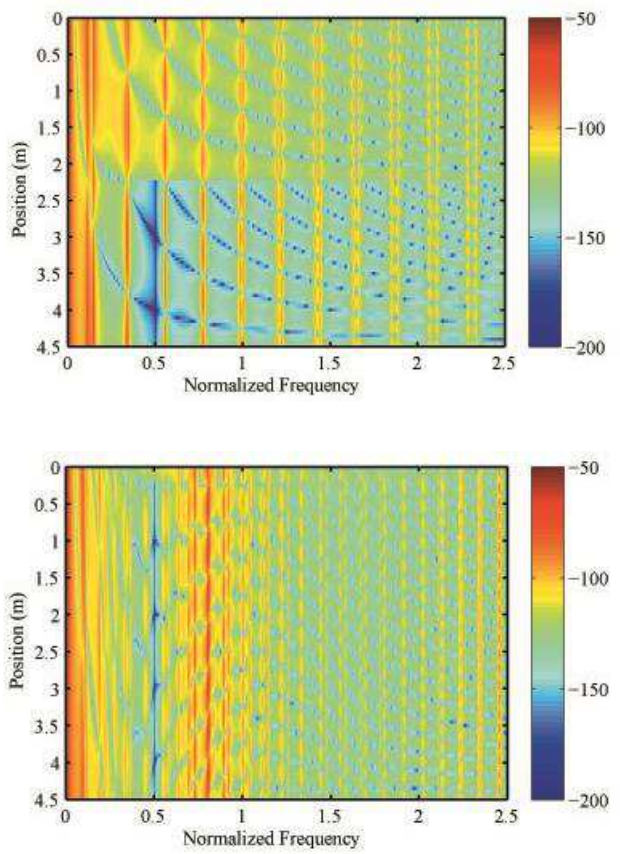

(b)
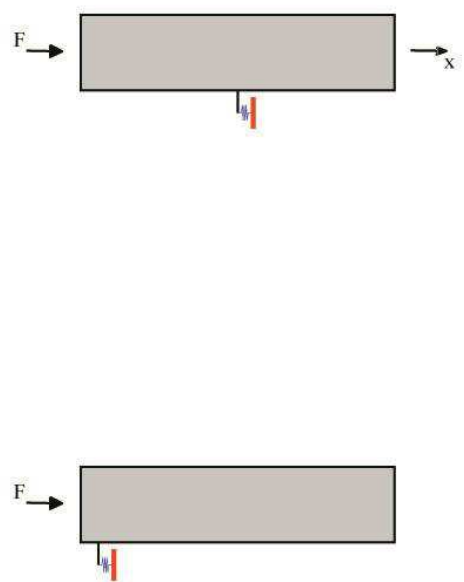

(c)

Fig. 5 2D maps of the FRF (in $\mathrm{dB}$ ) of the homogeneous rod with one single vibration absorber: (a) mass fraction of $8 \%$, location at $x=L / 2$; (b) mass fraction of $44 \%$, location at $x=L / 2$; (c) mass fraction of $14 \%$, location at $x=\Delta / 2$ 


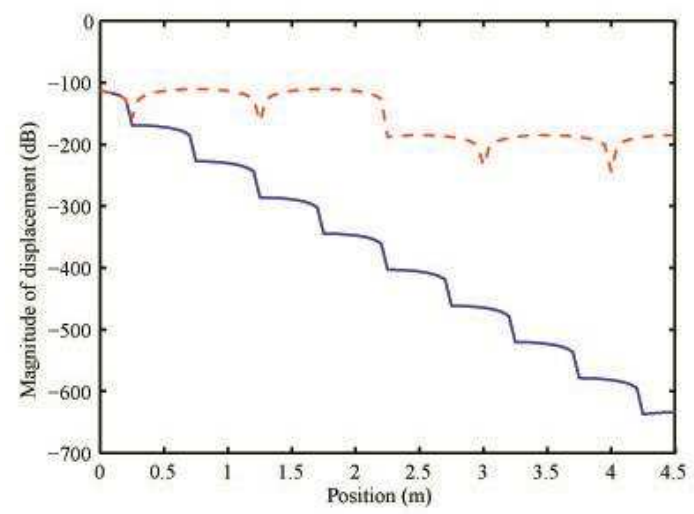

Fig. 6 Spatial variations of the reduction of the vibration levels at $f=0.5 f_{\text {ref }}=790.6 \mathrm{~Hz}$ : (blue color) periodic array of resonant devices with 9 resonators and mass fraction of $44 \%$, (red color) vibration absorber located at $x=\Delta / 2$ and with mass fraction of $44 \%$

interesting feature when using a periodic array of resonant devices is that it generates band gaps with global attenuation of the vibration levels (i.e., in frequency and space) along the structure. By comparing resonant devices of different masses to each other, one may notice that higher mass fractions produce higher levels of attenuation and larger attenuation zones (see Figs. 4(b)-(c)).

Consider now the 2D maps of the FRFs of the rod with one single vibration absorber. The results are shown in Fig. 5 for different mass fractions (8\%, 44\% and 14\%) and positions of the vibration absorber $(x=L / 2$ and $x=\Delta / 2)$. Those results are issued from a conventional analytical approach that consists in modeling each rod component (before and after the resonator) by means of a spectral element (Doyle 1997) -i.e., by modeling its dynamic stiffness matrix by means of two longitudinal waves propagating in right and left directions, respectively-and modeling the resonator by means of a spring-mass system. As it can be seen, band gaps formed by Bragg scattering mechanisms-i.e., for $f / f_{\text {ref }}=1$ and $f / f_{\text {ref }}=2$-are not observed in any case, as opposed to the periodic array of resonant devices even though in case when the added masses involved are the same. Also, the attenuation zone remains restricted to a short frequency bandwidth around $f / f_{\text {ref }}=0.5$. Notice that the attenuation zone is local, i.e., concentrated at anti-resonance points. Moreover, it depends on the locations of the vibration absorber and excitation point on the structure. In contrast, due to their global attenuation effect, periodic arrays of resonant devices have the potentiality to be used for excitations located at arbitrary locations, i.e., which are not necessarily known. This feature is clearly highlighted in Fig. 6 in which the spatial variations in the reduction of the vibration levels produced, respectively, by the vibration absorber and a periodic array of resonant devices of same masses, are plotted at the target resonance frequency $f=0.5 f_{\text {ref }}$.

\section{Band gap generation in a 3D aircraft fuselage-like structure}

\subsection{Problem description}

In this section, one aims at showing that important reduction of the vibration levels of complex 
periodic structures can be achieved through the use of a lightweight periodic array of resonant devices whose parameters are adequately tuned. The periodic structure that is considered here represents a coarse approximation of a 3D aircraft fuselage, consisting in a thin cylindrical shell with axial stiffeners (stringers) and circumferential stiffeners (frames), see Fig. 7. Those stiffeners are periodically distributed over the cylinder, two consecutive circumferential (resp. axial) stiffeners being spaced of $0.4 \mathrm{~m}$ (resp. of $11.25^{\circ}$ ). The material and geometric characteristics of the stiffened cylindrical shell are listed in Table 2.

Within the framework of the WFE method (Section 2.1), a substructure is considered as shown in Fig. 7(b), which consists in a quarter of the stiffened cylindrical shell with a length $\Delta=0.4 \mathrm{~m}$. Symmetry boundary conditions are considered so as to model the structure along the whole circumference. Regarding the substructure, the cylinder and stiffeners are meshed by means of

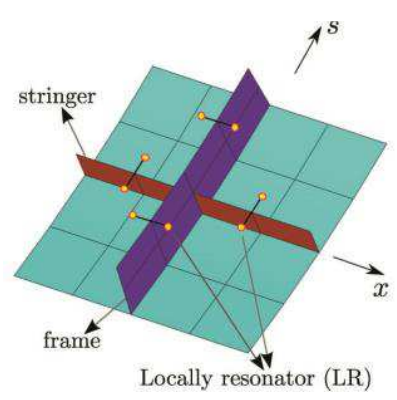

(a)

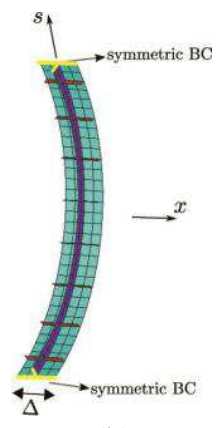

(b)

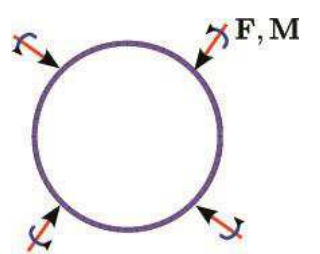

Front view

(c)

Fig. 7 FE model of the 3D aircraft fuselage-like structure: (a) unit cell; (b) substructure; (c) whole structure

Table 2 Characteristics of the stiffened cylindrical shell.

\begin{tabular}{cccc}
\hline \hline Parameter & cylindrical shell & frames & stringers \\
\hline Thickness $(t)$ & $0.001 \mathrm{~m}$ & $0.0012 \mathrm{~m}$ & $0.005 \mathrm{~m}$ \\
Radius $(R)$ & $2 \mathrm{~m}$ & - & - \\
Height $(h)$ & - & $0.10 \mathrm{~m}$ & $0.05 \mathrm{~m}$ \\
Density $(\rho)$ & & $2700 \mathrm{~kg} / \mathrm{m}^{3}$ & \\
Young's Modulus $(E)$ & $70 \mathrm{GPa}$ & \\
Poisson's ratio $(v)$ & 0.3 & \\
Loss factor $(\eta)$ & 0.01 & \\
\hline
\end{tabular}


Table 3 Characteristics of the resonant devices

\begin{tabular}{ccc}
\hline \hline Parameter & mounted on frames & mounted on stringers \\
\hline Beam cross-section Area $\left(A_{b}\right)$ & $5.435910^{-6} \mathrm{~m}^{2}$ & $7.587810^{-6} \mathrm{~m}^{2}$ \\
Beam length $\left(l_{b}\right)$ & $0.03 \mathrm{~m}$ \\
Beam density $\left(\rho_{b}\right)$ & $1400 \mathrm{~kg} / \mathrm{m}^{3}$ \\
Beam Young's Modulus $\left(E_{b}\right)$ & $70 \mathrm{GPa}$ \\
Beam Poisson's ratio $\left(v_{b}\right)$ & 0.3 \\
Beam loss factor $\left(\eta_{b}\right)$ & 0.01 \\
Concentrated mass $\left(m_{r}\right)$ & $0.0125 \mathrm{~kg}$ \\
\hline
\end{tabular}

quadrilateral Reissner-Mindlin shell elements with six DOFs per node, i.e., translations in the $x, y$ and $z$ directions and rotations about the same axes. Here, the total number of DOFs used to discretize the substructure is 1,578 , with $246 \mathrm{DOF}$ over the left/right boundary and 1,086 internal DOFs. The whole periodic structure is modeled by means of $N=40$ identical substructures connected along the $x$-direction, as shown in Fig. 7(c). It is excited by unitary harmonic radial and axial forces, as well as moments, which are applied on the left end at $\theta=45^{\circ}$, and it is free from excitations on its right end.

Periodic arrays of resonant devices are considered so as to attenuate the vibration levels throughout the whole structure. The motivation behind the present study is to design lightweight resonant devices which may be effectively attached to a real aircraft fuselage. In the present study, each resonant device is modeled as a flexural Euler beam with a concentrated mass on one end and whose other end is connected to the stringers and frames of the periodic structure at the other end, as shown in Fig. 7(a). The first flexural resonances of those resonators are assigned to be $197 \mathrm{~Hz}-$ which corresponds to one resonance frequency of the whole periodic structure -and $275 \mathrm{~Hz}$, respectively. The characteristics of the resonators are listed in Table 3. Notice that the added mass induced by the periodic array of these resonant devices represents about $12.5 \%$ of the total mass to the original periodic structure, i.e., it remains small. Within the WFE framework, a substructure is thus considered which takes into account the FE models of the resonant beams along with those of the cylinder, frames and stringers. This yields 3,114 DOFs for modeling the substructure, with 246 DOFs over the left/right boundary, and 2,622 internal DOFs. By considering the symmetry boundary conditions, this yields $n=240$ right-going and left-going wave modes which are to be computed by means of the WFE method.

\subsection{Numerical analysis and discussion}

The dispersion curves of the periodic structure are assessed by means of the WFE method as shown in Fig. 8. Regarding Fig. 8(a) (without resonant devices), it should be noticed that one particular wave mode which propagates along the 3D aircraft fuselage-like structure becomes evanescent between $230 \mathrm{~Hz}$ and $240 \mathrm{~Hz}$, which characterizes a band gap phenomenon. This might be explained by the fact that the circumferential stiffeners (frames) exhibit some flexural vibration modes in this frequency band. Regarding Fig. 8(b) (with resonant devices), it is seen that the added periodic array of resonant devices yields large evanescent parts around the target frequencies 197 $\mathrm{Hz}$ and $275 \mathrm{~Hz}$, which means that the propagation of waves is strongly attenuated.

Also, 2D maps of the FRFs of the periodic structure are shown in Fig. 9. Here, the root mean 


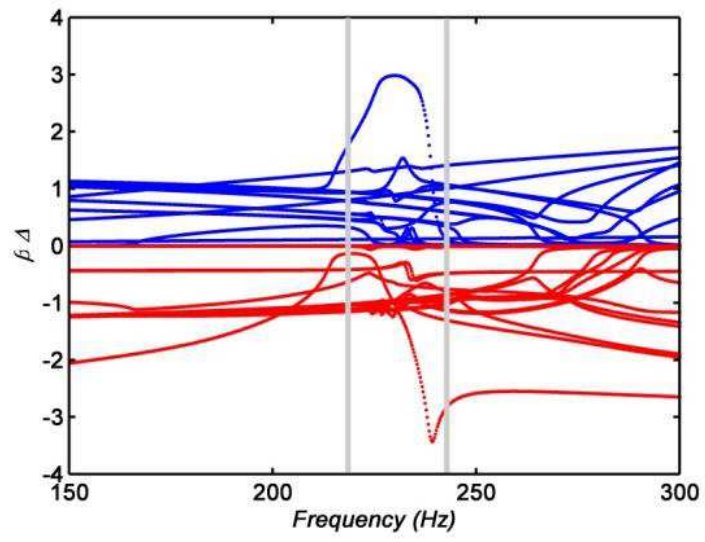

(a)

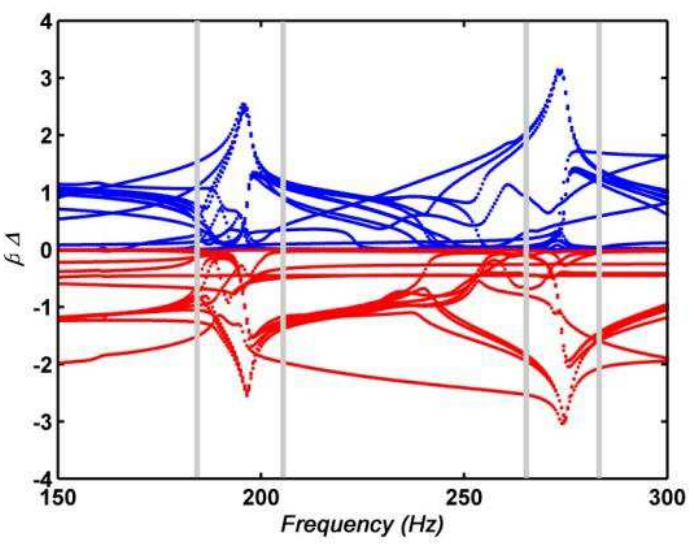

(b)

Fig. 8 Dispersion curves of the waves traveling along the 3D aircraft fuselage-like structure: (a) without resonant devices; (b) with the periodic array of resonant devices. (blue color) real part of $\beta_{j} \Delta$; (red color) imaginary part of $\beta_{j} \Delta$

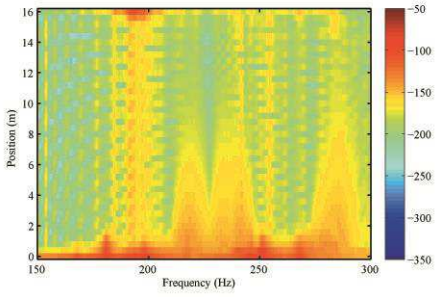

(a)

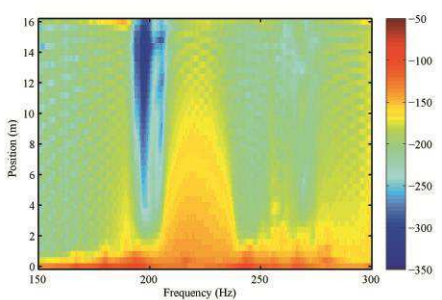

(d)

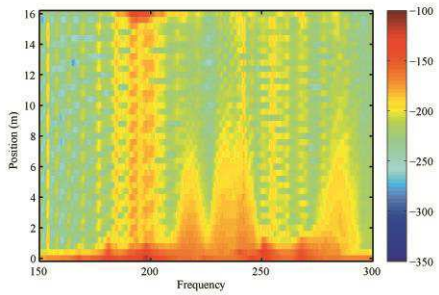

(b)

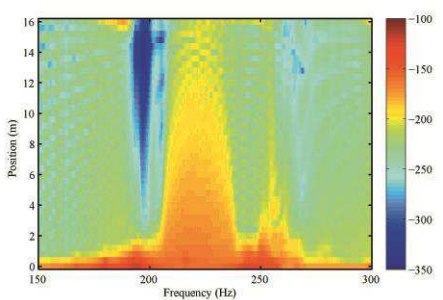

(e)

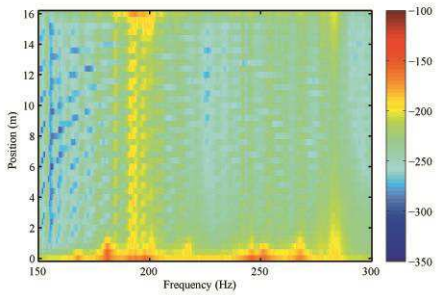

(c)

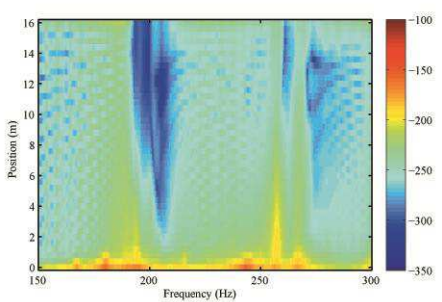

(f)

Fig. 9 RMS levels of the radial $(\mathrm{a}, \mathrm{d})$, tangential $(\mathrm{b}, \mathrm{e})$ and axial $(\mathrm{c}, \mathrm{f})$ displacements of the structure without devices (top) and with resonant devices (bottom)

square (RMS) levels of the radial, tangential and axial displacements (over each cross-section between two consecutive substructures) are displayed as functions of the frequency and position along the structure. As it can be seen, the consideration of the periodic array of resonant devices 


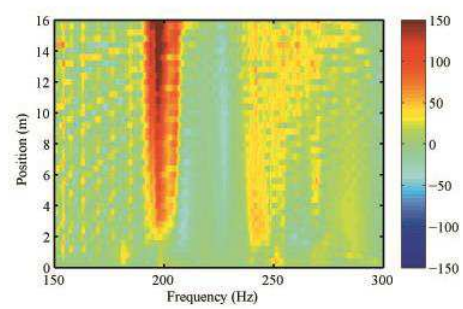

(a)

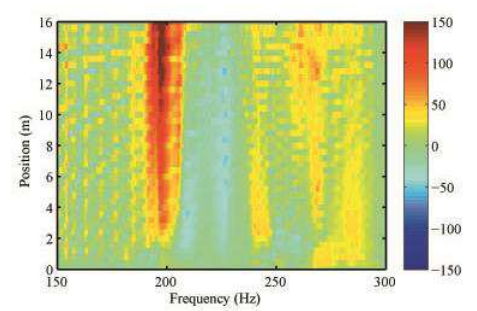

(b)

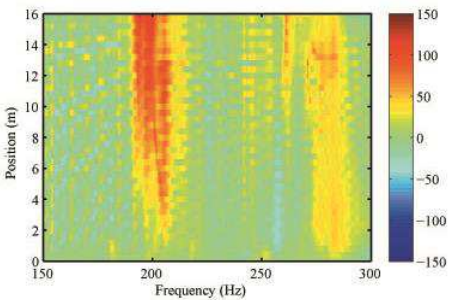

(c)

Fig. 10 Attenuation between the FRFs of the controlled and uncontrolled structure (in dB): (a) radial displacements; (b) tangential displacements; (c) axial displacements

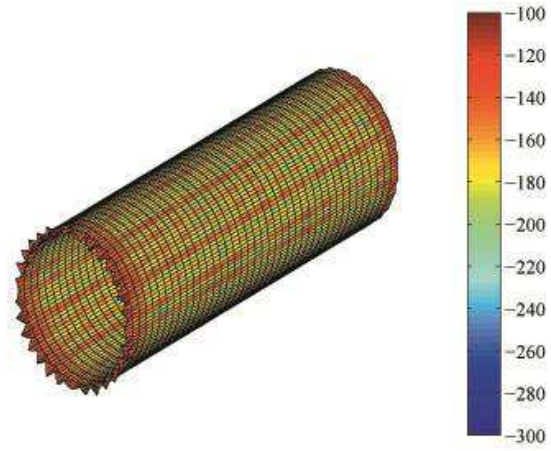

(a)

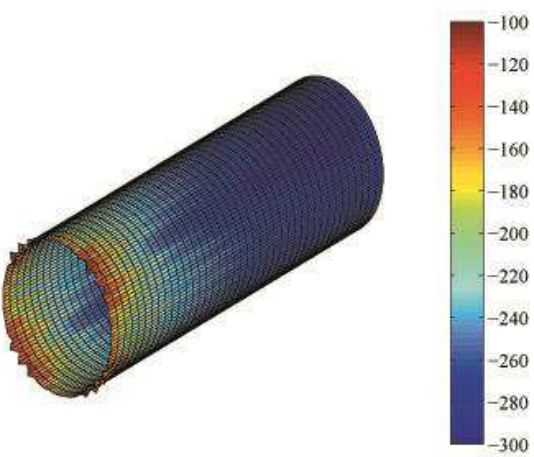

(b)

Fig. 11 Spatial distribution of the total displacement of the periodic structure (in $\mathrm{dB}$ ), at $275 \mathrm{~Hz}$ : (a) without resonant devices; (b) with resonant devices

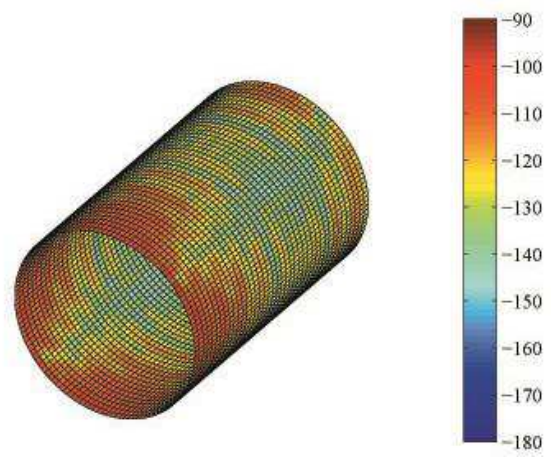

(a)

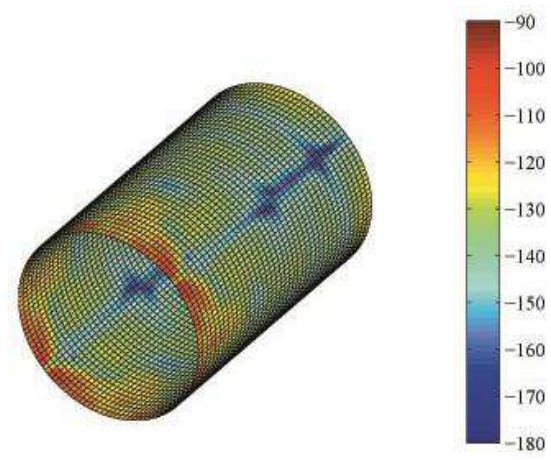

(b)

Fig. 12 Spatial distribution of the axial displacement of the periodic structure (in $\mathrm{dB}$ ), at $275 \mathrm{~Hz}$ : (a) without resonant devices; (b) with resonant devices

induces a large decrease of the vibration levels around the target frequency $197 \mathrm{~Hz}$, as expected. Vibration decrease is also observed around the other target frequency $275 \mathrm{~Hz}$, even though it does 
not correspond to a resonance frequency of the periodic structure. The attenuation between the FRFs of the controlled and uncontrolled structure are plotted in Fig. 10 in dB. Such a decrease in the vibration levels appears to be widely spread over the length of the structure, even near the excited cross-section. This phenomenon is clearly highlighted in Figs. 11-12, which highlight the spatial distributions of the displacements at $197 \mathrm{~Hz}$ and $275 \mathrm{~Hz}$, respectively. This provides a clear evidence that the magnitude of the whole displacement field of the structure can be strongly decreased.

It is worth pointing out that the proposed WFE approach makes use of full wave mode bases for modeling periodic structures, i.e., it does not invoke any basis truncation or reduction of the interface DOFs. In other words, the WFE method provides, in theory, the same level of accuracy as the FE method for modeling periodic structures. However, the WFE method can greatly improve the computational efficiency of the FE method because it involves modeling a single substructure. Further evidence of the efficiency of the WFE method is shown by comparing the sizes of the FE and WFE matrix systems. Considering the present case, the corresponding FE model of the structure with resonant devices would have involved 114,966 DOFs, compared to 3,144 DOFs with the WFE method. This fully gives credit to the proposed approach.

\section{Conclusions}

In this work, a passive vibration control of periodic structures has been analyzed which involves considering periodic arrays of simple resonant devices. The WFE method has been used for this purpose which constitutes a fast and efficient means for assessing the dispersion curves and FRFs of periodic structures. It has been shown that the consideration of lightweight periodic arrays of resonant devices yields large and global decreases of the vibration levels, even at low frequencies. Such resonant devices have proved to be relevant for treating large-sized complex 3D structures such as aircraft fuselages. Future work may involve those resonant devices for passive control of internal acoustics in fluid-filled periodic structures.

\section{Acknowledgments}

The authors are grateful to the government funding agency Fundação de Amparo à Pesquisa de São Paulo, FAPESP, for the financial support provided for the present research work through processes 2010/17317-9 and 2013/23542-3. The authors are also thankful to the Program "Cátedras Francesas na UNICAMP" and the National Research Council, CNPq, through process 304482/2011-0.

\section{References}

Bennett, M. S. and Accorsi, M. L. (1994), "Free wave propagation in periodically ring stiffened cylindrical shell", J. Sound Vib., 171(1), 49-66.

Doyle, J. F. (1997), Wave Propagation in Structures, 2nd Edition, Springer-Verlag, New York, NY, USA.

Goffaux, C., Sánchez-Dehesa, J., Yeyati, A. L., Lambin, Ph., Khelif, A., Vasseur, J. O. and Djafari-Rouhani, B. (2002), "Evidence of Fano-like interference phenomena in locally resonant materials", Phys. Rev. Lett., 
$\mathbf{8 8}(22), 225502$.

Goldstein, A.L., Silva, P.B. and Arruda, J.R.F. (2010), "The wave spectral finite element method applied to the design of periodic waveguides", Proceedings of the 18th International Congress on Sound and Vibration, Rio de Janeiro, Brazil, July.

Kashina, V.I. and Tyutekin, V.V. (1990), "Waveguide vibration reduction of longitudinal and flexural modes by means of a multielement structure of resonators", Sov. Phys. Acoust., 36, 383-385.

Kushwaha, M.S., Halevi, P., Dobrzynski, L. and Djafari-Rouhani, B. (1993), "Acoustic band structure of periodic elastic composites", Phys. Rev. Lett., 71(13), 2022-2025.

Kushwaha, M.S., Halevi, P., Martinez, G., Dobrzynski, L. and Djafari-Rouhani, B. (1994), "Theory of acoustic band structure of periodic elastic composites", Phys. Rev. B., 49(4), 2313-2322.

Lee, S., Vlahopoulos, N. and Waas, A.M. (2010), "Analysis of wave propagation in a thin composite cylinder with periodic axial and ring stiffeners using periodic structure theory", J. Sound Vib., 329(16), 3304-3318.

Liu, Z., Zhang, X., Mao, Y., Zhu, Y.Y., Yang, Z., Chan, C.T. and Sheng, P. (2000), "Locally resonant sonic materials", Science, 289(5485), 1734-1736.

Mencik, J.M. (2010), "On the low-and mid-frequency forced response of elastic structures using wave finite elements with one-dimensional propagation”, Comput. Struct., 88(11-12), 674-689.

Mencik, J.M. (2014), "New advances in the forced response computation of periodic structures using the wave finite element (WFE) method", Comput. Mech., 54(3), 789-801.

Mencik, J.M. and Ichchou, M.N. (2005), "Multi-mode propagation and diffusion in structures through finite elements", Eur. J. Mech. A/Solid., 24(5), 877-898.

Ormondroyd, J. and den Hartog, J.P. (1928), "The theory of the dynamic vibration absorber", T. Am. Soc. Mech. Eng., 50, A9-A22.

Sigalas, M.M. and Economou, E.N. (1992), "Elastic and acoustic wave band structure", J. Sound Vib., 158(2), 377-382.

Sigalas, M.M., Kushwaha, M.S., Economou, E.N., Kafesaki, M., Psarobas, I.E. and Steurer, W. (2005), "Classical vibrational modes in phononic lattices: theory and experiment", Z. Kristallogr., 220, 765-809.

Silva, P.B., Mencik, J.M. and Arruda, J.R.F. (2014), "On the forced harmonic response of coupled systems via a WFE-based super-element approach", Proceedings of the International Conference on Noise and Vibration Engineering (ISMA), Leuven, Belgium, September.

Silva, P.B., Mencik, J.M. and Arruda, J.R.F. (2015), "Wave finite element based super-elements for forced response analysis of coupled systems via dynamic substructuring”, Int. J. Numer. Meth. Eng., doi: $10.1002 /$ nme.5176.

Sorokin, S.V. and Ershova, O.A. (2004), "Plane wave propagation and frequency band gaps in periodic plates and cylindrical shells with and without heavy fluid loading", New J. Phys., 278(3), 501-526.

Thompson, D.J. (2008), "A continuous damped vibration absorber to reduce broad-band wave propagation in beams", J. Sound Vib., 311, 824-842.

Wang, Z., Zhang, P. and Zhang, Y. (2013), "Locally resonant band gaps in flexural vibrations of a Timoshenko beam with periodically attached multioscillators", Math. Probl. Eng., 2013 (Article ID 146975), 10.

Xiao, Y., Mace, B.R., Wen, J. and Wen, X. (2011), "Formation and coupling of band gaps in a locally resonant elastic system comprising a string with attached resonators", Phys. Lett. A, 375, 1485-1491.

Xiao, Y., Wen, J. and Wen, X. (2012), "Longitudinal wave band gaps in metamaterial-based elastic rods containing multi-degree-of-freedom resonators", New J. Phys., 14, 033042.

Xiao, Y., Wen, J., Yu, D. and Wen, X. (2013), "Flexural wave propagation in beams with periodically attached vibration absorbers: Band-gap behavior and band formation mechanisms", J. Sound Vib., 332, 867-893.

Zhong, W.X. and Williams, F.W. (1995), "On the direct solution of wave propagation for repetitive structures", J. Sound Vib., 181(3), 485-501. 